\title{
Effects of repeated consumption on sensory-enhanced satiety
}

\author{
Martin R. Yeomans ${ }^{1 *}$, Keri McCrickerd ${ }^{1}$, Jeffrey M. Brunstrom ${ }^{2}$ and Lucy Chambers ${ }^{1}$ \\ ${ }^{1}$ School of Psychology, University of Sussex, Brighton BN1 9QH, UK \\ ${ }^{2}$ School of Experimental Psychology, University of Bristol, Bristol BS8 1TU, UK \\ (Submitted 5 March 2013 - Final revision received 14 August 2013 - Accepted 14 August 2013 - First published online 12 November 2013)
}

\section{Abstract}

Previous research has suggested that sensory characteristics of a drink modify the acute satiating effects of its nutrients, with enhanced satiety being evident when a high-energy drink was thicker and tasted creamier. The present study tested whether this modulation of satiety by sensory context was altered by repeated consumption. Participants $(n$ 48) consumed one of four drinks mid-morning on seven non-consecutive days, with satiety responses being measured pre-exposure (day 1), post-exposure (day 6) and at a 1-month follow-up. The drinks combined two levels of energy (lower energy (LE), $326 \mathrm{~kJ}$ and higher energy, $1163 \mathrm{~kJ}$ ) with two levels of satietypredictive sensory characteristics (low sensory (LS) or enhanced sensory). Test lunch intake 90 min after drink consumption depended on both the energy content and sensory characteristics of the drink before exposure, but on the energy content alone after exposure and at the follow-up. The largest change was an increase in test meal intake over time in the LE/LS condition. The effects on intake were reflected in appetite ratings, with rated hunger and expected filling affected by sensory characteristics and energy content preexposure, but were largely determined by energy content post-exposure and at the follow-up. In contrast, a measure of expected satiety reflected sensory characteristics regardless of energy content on all the three test days. Overall, these data suggest that some aspects of the sensory modulation of satiety are changed by repeated consumption, with covert energy becoming more effective in suppressing appetite over time, but also suggest that these behavioural changes are not readily translated into expectations of satiety.

Key words: Satiety: Expectations: Flavour

Although there is considerable evidence that the post-ingestive physiological effects of nutrient intake generate a series of signals that contribute to satiety ${ }^{(1-3)}$ (a distinction can be made between how effective a food is at suppressing appetite while it is being consumed (satiation) and during the period after it has been ingested (satiety)), a model of satiety based on gastrointestinal signalling alone fails to fully explain differences in satiety between products. For example, nutrients ingested as beverages often lead to weak satiety ${ }^{(4)}$, yet similar nutrients ingested as soup generate much stronger satiety ${ }^{(5)}$. One explanation for such discrepancies is that information present at the time of consumption generates expectations that modulate post-ingestive satiety processes, and the overall experience of satiety reflects this integration of cognitive, sensory and nutrient-induced cues. An increasing number of studies have supported this view ${ }^{(6-9)}$. Thus, altering the sensory characteristics of a drink to give it a slightly thicker texture and more creamy flavour both generated expectations that the product would be more satiating ${ }^{(10)}$ and resulted in increased satiety when consumed in combination with additional energy, indexed both from the ratings of appetite post-ingestion and intake at a test meal ${ }^{(7,8)}$. Beliefs about the likely effect of the ingested food or drink do not just alter the behavioural responses, however. First, when participants consumed a solid (gel), or believed that a liquid would turn to a gel in their stomach, they reported greater satiety and showed larger increases in insulin and glucagon-like peptide 1 than when the same nutrients were consumed as a drink or as a gel with the expectation that the gel would become liquid ${ }^{(9)}$ Likewise, ingestion of a product labelled as indulgent produced a steeper decline in the hunger hormone ghrelin than when labels suggested a low-energy milkshake ${ }^{(11)}$.

Studies of cognitive and sensory influences on satiety to date have concentrated on acute effects, and a key question is whether such effects are maintained following repeated exposure. According to learned satiety ${ }^{(12)}$, repeated co-experience of the sensory characteristics of the consumed product and subsequent experience of satiety should lead to more accurate appetite regulation with experience, evidenced either by more accurate compensation at the test meal ${ }^{(13,14)}$ or by changes in the expectations that the drink will be satiating ${ }^{(15)}$. Although evidence for learned satiety from studies of

Abbreviations: ES, enhanced sensory; HE, high energy; LE, low energy; LS, low sensory.

*Corresponding author: Professor M. R. Yeomans, fax + 441273 678058, email martin@sussex.ac.uk 
repeated consumption is weak ${ }^{(16)}$, two studies have suggested that this was possible here. First, people's expectations about how satiating a product was changed in line with actual nutrient content after just one exposure ${ }^{(17)}$, although a subsequent study using similar methodology but longer exposure has found no such effects ${ }^{(18)}$. Second, there was stronger evidence of learned satiety (indexed by a decrease in ad libitum consumption over time) when a drink's textural (viscosity) rather than flavour cues predicted nutrient content ${ }^{(19)}$, perhaps because texture is a more consistent predictor of energy $^{(20)}$. Thus, learned satiety might be more evident after repeated exposure to a high-energy product with sensory characteristics that predict satiety than after exposure to the same product without these sensory characteristics. Building on methodology from studies of sensory modulation of nutrient-based satiety ${ }^{(7,8)}$, we tested this prediction by repeatedly exposing participants to low- or high-energy beverage preloads with or without added thick and creamy sensory properties and by measuring the effects on expected and actual satiety responses.

\section{Methods}

\section{Study design}

The satiating effects of one of four versions of a test drink combining two levels of energy (lower energy (LE), $326 \mathrm{~kJ}$ and higher energy (HE), $1163 \mathrm{~kJ}$ ) with two levels of satietypredictive sensory characteristics (low sensory (LS) or enhanced sensory (ES)) were measured at the start of testing (preexposure, day 1), after four exposure days (post-exposure, day 6) and 1 month later (follow-up, day 7).

\section{Participants}

A total of forty-eight non-obese (mean BMI 23.6 (range $19-30) \mathrm{kg} / \mathrm{m}^{2}$ ) young (age mean $21 \cdot 3$ (range $18-34$ ) years) men participated, mostly undergraduates at the University of Sussex. Volunteer men whose details on a recruitment database suggested that they were unrestrained (Three-Factor Eating Questionnaire Restraint score $\leq 8^{(21)}$ ) and who selfreported smoking less than five cigarettes per week were told that the purpose of the study was 'To investigate how a mid-morning snack influences your mood'. Respondents who confirmed that they were generally healthy, were not taking any prescription medication and were not allergic or

Table 1. Age, BMl and restraint scores for the four groups of participants (Mean values with their standard errors, $n$ 12)

\begin{tabular}{lcccccccc}
\hline & \multicolumn{2}{c}{ Age (years) } & & \multicolumn{2}{c}{ BMI $\left(\mathrm{kg} / \mathrm{m}^{2}\right)$} & & \multicolumn{2}{c}{ Restraint } \\
\cline { 2 - 3 } Drink conditions & Mean & SEM & & Mean & SEM & & Mean & \multicolumn{2}{c}{ SEM } \\
\hline LE/LS & 21.6 & 0.7 & & 24.4 & 0.9 & & 3.8 & 0.5 \\
LE/ES & 19.2 & 0.3 & & 22.9 & 0.7 & & 2.1 & 0.5 \\
HE/LS & 21.2 & 0.5 & & 24.9 & 1.3 & & 3.1 & 0.5 \\
HE/ES & 23.3 & 1.3 & & 23.3 & 0.7 & & 4.3 & 0.7 \\
\hline
\end{tabular}

LE, low energy; HE, high energy; LS, low sensory; ES, enhanced sensory. aversive to any of the foods and ingredients used in the study were assigned at random to one of the four treatment conditions, and these four groups did not differ significantly in age or BMI (Table 1). The present study was conducted according to the guidelines laid down in the Declaration of Helsinki (1996), and was approved by the University of Sussex Ethics Committee. Written informed consent was obtained from all participants.

\section{Test foods}

Breakfast. On each day, participants consumed a set breakfast (total $1678 \mathrm{~kJ})$, consisting of cereal ( $60 \mathrm{~g}$, Crunchy Nut Cornflakes; Kellogg's), semi-skimmed milk (160 g; Sainsbury's) and orange juice (200 g; Sainsbury's).

Beverage preload. Test beverages were $320 \mathrm{~g}$ portions of mango- and peach-flavoured yogurt drinks, served in commercial 'smoothie' bottles (Esterform). In total, four versions were developed, two LE (326 kJ) and two HE (1163 kJ), with energy content manipulated by adding maltodextrin (C*PUR 1910; Cargill) with either LS or ES characteristics (sensory enhancements achieved by adding tara gum (Kalys), milk caramel flavour (S Black) and vanilla extract (NeilsenMassey)) based on previous studies which confirmed that LE and HE drinks were sensorially similar, and ES drinks were thicker and creamier than LS drinks ${ }^{(7,8,10)}$. The full ingredients were as follows: mango juice (all versions: $100 \mathrm{~g}$; Tropicana); peach squash drink (all versions: 35 g; Robinson's); 0\% fat fromage frais (LE versions: $55 \mathrm{~g}$; HE versions: $30 \mathrm{~g}$; Sainsbury's); water (LE versions: $130 \mathrm{~g}$; HE versions: $100 \mathrm{~g}$ ); maltodextrin (HE versions: $55 \mathrm{~g}$ ); yellow colour (LE versions: eight drops; Silverspoon); red colour (all versions: two drops; Silverspoon); tara gum (LE/LS: 0.3 g; LE/ES: $1.2 \mathrm{~g}$; HE/ES: $1 \mathrm{~g}$; Kalys); aspartame (LE versions: 0.03 g; Ajinomoto); vanilla extract (all ES versions: $1 \mathrm{~g}$ ); milk caramel flavour (all ES versions: $0.5 \mathrm{~g}$ ).

Test lunch. The satiety test included an ad libitum twocourse lunch consisting of pasta (each serving $250 \mathrm{~g}$ of cooked pasta, 'Conchiglie' (Sainsbury's), plus $250 \mathrm{~g}$ of tomato and basil pasta sauce (Sainsbury's)) followed by ice cream ('Chocolate Inspiration', Carte D'OR; Unilever). Participants were permitted to consume water ad libitum during this meal.

\section{Procedure}

Testing took place on seven non-consecutive weekdays at the Sussex Ingestive Behaviour Unit, UK. Satiety responses to the beverages were assessed at the start of testing (pre-exposure, day 1), after repeated consumption (post-exposure, day 6) and at the 1-month follow-up (day 7), with test days 2-5 serving as beverage exposure days. Test days 1-6 were conducted over a 3- to 4-week period, with each session separated by at least $1 \mathrm{~d}$; the final follow-up test took place at least 1 month after the post-exposure session. On all days, participants consumed breakfast in the laboratory between 08.45 and 09.45 hours, having consumed only water from 23.00 hours the previous evening. After breakfast, they were 
permitted to leave the laboratory, but could consume only water until they returned $2 \mathrm{~h}$ later.

On their return, participants evaluated their mood and appetite (baseline ratings) using Sussex Ingestion Pattern Monitor software (SIPM version 2.011, University of Sussex ${ }^{(22)}$, run on a personal computer). In line with the guise that the study examined the effects of the test drink on mood, participants rated their nervousness, clearheadedness, tiredness, happiness, alertness, nausea as well as hunger and fullness using visual analogue scales in the format of 'How < target rating $>$ do you feel right now?', end-anchored with 'Not at all $<$ target rating $>$ ' and 'Extremely $<$ target rating $>$ ', and in a randomised order. Only ratings of hunger and fullness were analysed.

Next, at the pre-exposure, post-exposure and follow-up sessions, participants completed an expected satiety task adapted from methodology developed by Brunstrom and colleagues $^{(23,24)}$. Expected satiety was defined as the anticipated suppression of hunger at the time after ingestion. Participants were presented with a sealed bottle of their beverage as an example of a standard portion plus a $20 \mathrm{ml}$ sample to be used for the task along with the instruction 'Take one mouthful of the sample of the yogurt drink in front of you. Imagine that you had consumed the whole bottle for your breakfast. Now imagine how hungry you would feel just before lunch. In this task, you will be asked to select the amount of breakfast cereal that you would need to eat to match the effect of the yogurt drink on your hunger'. They then adjusted the size of portions of cereal displayed on-screen to match their expectations about how much the yogurt beverage would suppress subsequent appetite. Seven cereal products that are well known by British consumers (Cocopops, Kellogg's; Branflakes, Kellogg's; Shreddies, Nestlé; Cheerios, Nestlé; Alpen, Weetabix; Crunchy Nut Clusters, Kellogg's; Cornflakes, Kellogg's) were used, with fifty photos of each cereal increasing logarithmically in portion size from 155 to $1904 \mathrm{~kJ}$. Then, onscreen instructions prompted participants to consume one mouthful of the beverage and then complete visual analogue scale ratings of its sweetness, creaminess, pleasantness, thickness, fillingness and familiarity using the same format as for the mood ratings. They were then allowed $10 \mathrm{~min}$ to consume their beverage, before re-rating mood and appetite (post-preload ratings). On the exposure-only sessions (days 2-5), participants were free to leave the laboratory, but were required to repeat mood and appetite questions (paper version) $90 \mathrm{~min}$ later, having consumed only water. At the pre-exposure, postexposure and follow-up sessions, participants returned to the laboratory $90 \mathrm{~min}$ later for their lunch session, having consumed only water.

The lunch session began with participants re-rating their mood and appetite (pre-lunch ratings). They were then served a portion of pasta, and they rated it for pleasantness, savouriness, saltiness and familiarity, before re-rating appetite (lunch appetiser ratings). Intake was covertly recorded by a balance (Model BP4200; Sartorius) built into the table and hidden underneath a place mat and connected to a personal computer running SIPM. Every time the participants consumed at least $400 \mathrm{~g}$ of pasta, an audible alert and on-screen message prompted the participants to call their researcher, who provided a new serving so that the participants could not use an empty bowl as a meal termination cue. Once the participants had eaten enough, they selected an on-screen button 'course completed'. Participants were then served $150 \mathrm{~g}$ of ice cream, which they rated for creaminess, sweetness, pleasantness and familiarity before consuming as much as they liked. Refills were provided whenever weight decreased by at least $100 \mathrm{~g}$. Lunch ended with participants selecting an on-screen button after which they re-rated appetite and mood (post-lunch ratings). Participants were paid $£ 40$ on completion of the post-exposure session and were invited to participate in the follow-up session, for which they were paid an additional £10. Height and weight were recorded at the end of testing followed by structured debriefing to record participants' beliefs about the purpose of the study.

\section{Data analysis}

The key questions were as follows: (1) did the degree to which the test drink generated expected and actual satiety depend on both its energy content and sensory characteristics, (2) were these effects modified by repeated consumption and (3) were these effects sustained 1 month later? To test the first two questions, measures of satiety (expected satiety, expected filling, changes in rated appetite post-consumption and intake at the test lunch) on the pre- and post-exposure days were contrasted using ANOVA, with energy density (LE $v$. HE) and sensory context (LS $v$. ES), both between participant, and test day (pre- or post-exposure, within participant) as factors. For expected satiety, where we had estimates of the amount $(\mathrm{kJ})$ of each of seven cereals that were expected to suppress hunger to the same extent as the drink, cereal type was included as a within-participant factor. For appetite ratings, initial analyses confirmed that there were no differences in hunger or fullness before drink consumption, allowing the calculation of changes from baseline immediately after consuming the drink, before lunch was served and after tasting the main course. These three rating times were included as a within-participant factor. As only forty-three participants completed the 1-month follow-up session, these data were analysed separately. Only one participant had a BMI greater than $30 \mathrm{~kg} / \mathrm{m}^{2}$, and therefore BMI was included as a covariate in all analyses.

\section{Results}

\section{Test lunch intake}

The analysis of total energy consumed at lunch (kJ; Fig. 1(a)) at the pre- and post-exposure sessions found a significant three-way interaction between the drink's energy content, sensory characteristics and test day $(F(1,43)=4.58, P=0.038$, $\left.\eta^{2}=0 \cdot 10\right)$, a significant main effect of energy content $\left(F(1,43)=14.73, P<0.001, \eta^{2}=0 \cdot 26\right)$ and a significant twoway interaction between energy content and test day $\left(F(1,43)=5 \cdot 11, P=0 \cdot 029, \eta^{2}=0 \cdot 11\right)$. These effects remained significant when only those participants who completed the 
(a)

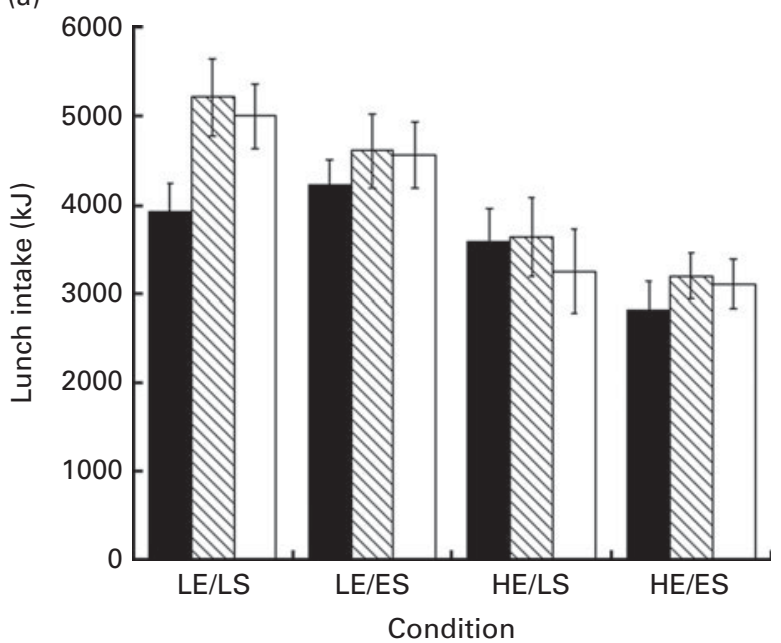

(b)

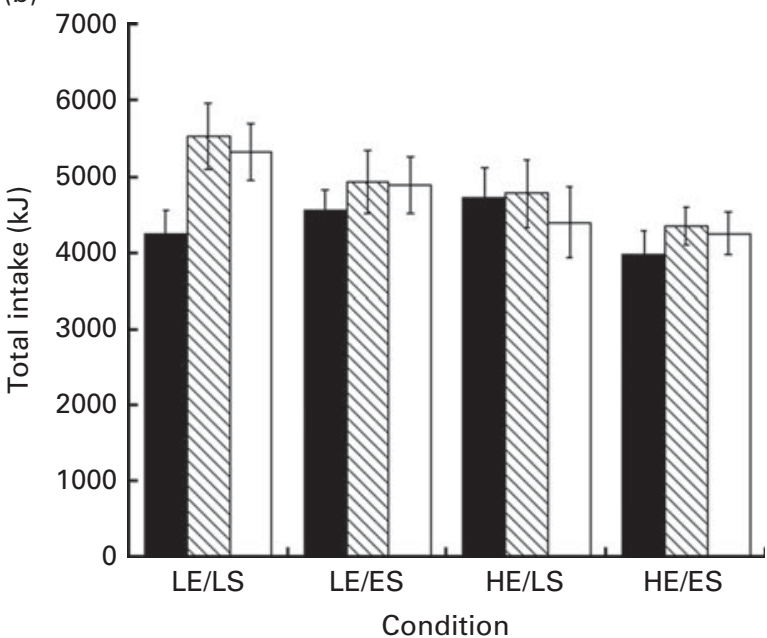

Fig. 1. Energy intake (kJ) both (a) at the test lunch and (b) as the sum of the test lunch and drink on the three test days (pre-exposure ( $\square$ ), post-exposure ( $\mathbb{\nabla}$ ) and follow-up ( $\square$ )), in the four drink conditions (LE, low energy; HE, high energy; LS, low sensory; ES, enhanced sensory). Values are means, with their standard errors represented by vertical bars. Pre- and post-exposure, $n 12$; follow-up LE/LS, $n 11$; follow-up LE/ES, $n$ 10; follow-up HE/LS, $n 11$; follow-up HE/ES, $n 11$.

follow-up session were included (three-way interaction between energy content, sensory characteristics and test day $\left(F(2,76)=3 \cdot 22, P=0 \cdot 046, \eta^{2}=0 \cdot 08\right)$, main effect of energy content $\left(F(1,38)=17 \cdot 46, \quad P<0.001, \quad \eta^{2}=0.32\right) \quad$ and test day $\times$ energy content interaction $(F(2,76)=3 \cdot 18, \quad P=0 \cdot 048$, $\left.\eta^{2}=0 \cdot 08\right)$ ).

To allow interpretation of the three-way interaction, follow-up ANOVA contrasted lunch intake $(\mathrm{kJ})$ in the four drink conditions on each day. At the pre-exposure session, significantly less amount was consumed at lunch in the HE/ES condition than in either LE condition, with the HE/LS condition being intermediate $(F(3,48)=3.92, \quad P=0.015$, $\left.\eta^{2}=0 \cdot 22\right)$. In contrast, at the post-exposure session, intake in the two LE conditions was significantly greater than that in both the HE conditions, but with no significant differences between the two HE conditions or the two LE conditions $\left(F(3,43)=5 \cdot 65, P=0 \cdot 002, \eta^{2}=0 \cdot 28\right)$. To further assess the effects of repeated consumption, lunch intake at the preand post-exposure sessions was contrasted within participants. The only significant change was an increase in intake in the LE/LS condition $\left(F(1,10)=4.68, P=0.049, \eta^{2}=0.08\right)$, although all the groups tended to eat more overall at the second test lunch. Likewise, at the 1-month follow-up, lunch intake still depended on which drink had been consumed $\left(F(3,43)=6.39, P=0.001, \eta^{2}=0.34\right)$, and intake in the two LE conditions was very similar, and significantly more than in both the HE conditions, which were also similar.

We also calculated total energy consumed (preload plus lunch energy; Fig. 1(b)). Total energy intake at the pre- and post-exposure sessions again depended on a combination of
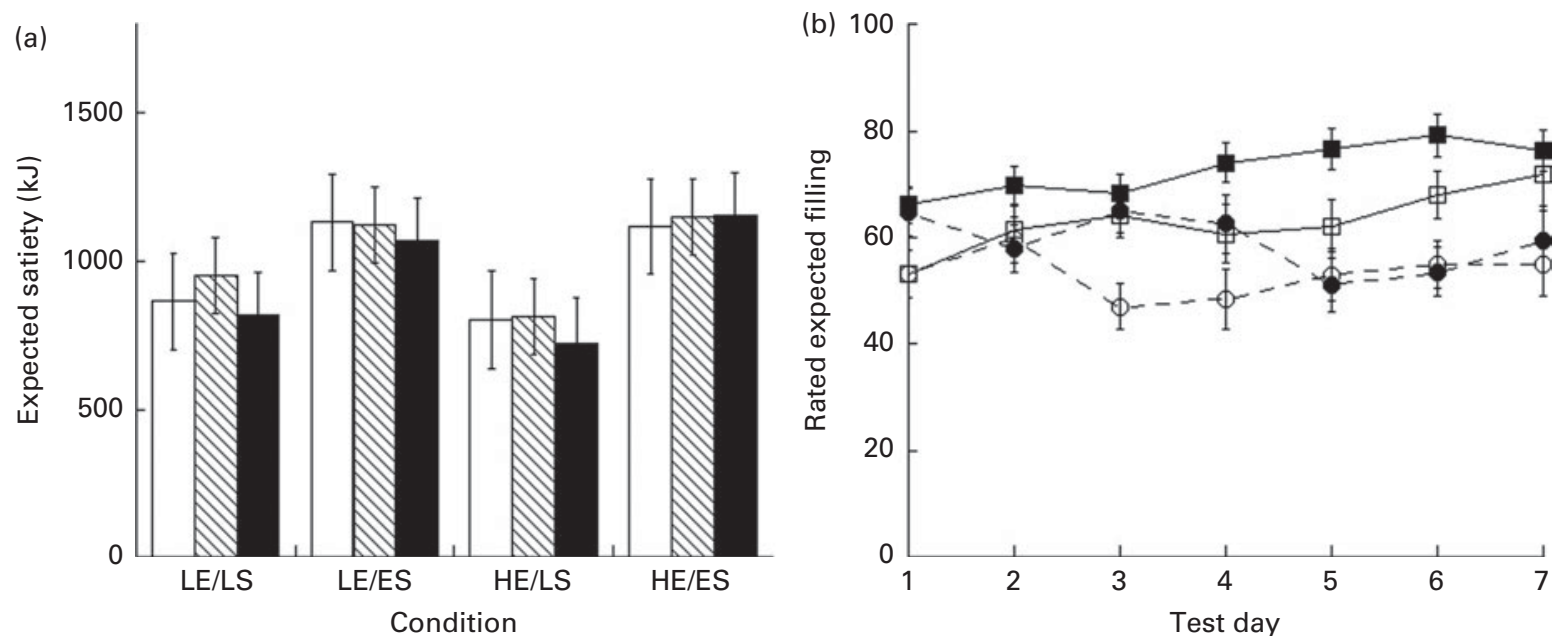

Fig. 2. (a) Expected satiety and (b) expected filling evaluations. (a) Estimated energy (kJ) of cereal needed to match predicted pre-lunch hunger in the four drink

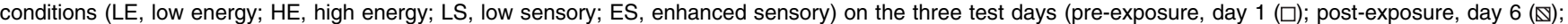
follow-up, day $7(\square)$ ). Values are means, with their standard errors represented by vertical bars. (b) Expected filling ratings on all $7 \mathrm{~d}$ : pre-exposure (day 1 ); $4 \mathrm{~d}$ of exposure (days 2-5); post-exposure (day 6); follow-up (day 7). Values are means, with their standard errors represented by vertical bars. Pre- and post-exposure, $n$ 12; follow-up LE/LS (--), $n$ 11; follow-up LE/ES (-๑), $n$ 10; follow-up HE/LS (־), $n 11$; HE/ES (-口), $n 11$. 
the drink's energy content, sensory characteristics and test day $\left(F(1,43)=5 \cdot 70, P=0 \cdot 021, \eta^{2}=0 \cdot 12\right)$. Separate analyses on each day found a marginally significant two-way interaction between energy content and sensory characteristics at the pre-exposure session $\left(F(1,43)=3.75, \quad P=0.06, \quad \eta^{2}=0.08\right)$, and a marginal main effect of energy content at the postexposure session $\left(F(1,43)=3.83, P=0.057, \eta^{2}=0.08\right)$, but there were no other significant main effects or interactions. Overall, total energy intake was least after consuming the HE/ES drink on both these days, and the effect of energy content at the post-exposure session confirms that repeated consumption increased the effects of energy manipulation and reduced the effects of sensory enhancements. However, the surprising finding was the relative overconsumption in the LE/LS condition after repeated exposure. Data from the follow-up session confirmed that participants consuming the HE drinks consumed significantly less in total than those consuming the LE drinks $(F(1,43)=4.91$, $\left.P=0 \cdot 033, \eta^{2}=0 \cdot 11\right)$, and, again, most was consumed in the LE/LS condition.

\section{Expected satiety and ratings of expected filling}

To calculate an overall measure of expected satiety, the average energy content $(\mathrm{kJ})$ of the portion of cereal judged to generate the same level of satiety as the test drink was determined from the seven cereal comparisons (Fig. 2(a)). These values varied depending on the sensory characteristics of the $\operatorname{drink}\left(F(1,43)=4 \cdot 81, P=0.034, \eta^{2}=0 \cdot 10\right)$ : participants consuming the ES drinks expected that they would need to eat more cereal to suppress hunger compared with those consuming the LS drinks. Expected satiety did not depend on energy content $\left(F(1,43)=0.15, P=0.70, \eta^{2}=0.01\right)$ nor was there any energy $\times$ sensory interaction $(F(1,43)=0.20, \quad P=0.66$, $\left.\eta^{2}=0 \cdot 01\right)$. There was also no evidence that expected satiety changed with exposure: the interaction between energy content, sensory context and test day was not significant $\left(F(1,43)=0 \cdot 14, P=0 \cdot 71, \eta^{2}=0 \cdot 01\right)$, nor was there any other significant interactions involving test day. The analysis of the 1-month follow-up data also found a significant effect of sensory characteristics on expected satiety $(F(1,38)=5 \cdot 34$, $\left.P=0.026, \eta^{2}=0 \cdot 12\right)$, but no other effects were significant. Thus, there was no evidence that the drink's energy content moderated expected satiety, or that repeated exposure led to changes in expected satiety.

Participants also rated how filling they expected the drink to be when they first tasted it on all days (Fig. 2(b)). The analysis of these ratings on days 1-6 found that expected filling varied with both energy content $(F(1,42)=$ 13.72, $\left.\quad P=0.001, \quad \eta^{2}=0.25\right)$ and sensory characteristics $\left(F(1,42)=7 \cdot 77, P=0 \cdot 008, \eta^{2}=0 \cdot 31\right)$, and also found a significant interaction between energy content and test day $\left(F(5,210)=2 \cdot 92, P=0.014, \eta^{2}=0 \cdot 07\right)$. At the pre-exposure session, expected filling only varied with sensory characteristics $\left(F(1,43)=8 \cdot 18, P=0 \cdot 007, \eta^{2}=0 \cdot 16\right)$, with the LS drink expected to be less filling than the ES drink. However, the ratings of expected filling increased over the $6 \mathrm{~d}$ in both the HE conditions, and decreased in the LE/ES condition. Consequently, at the post-exposure session, expected filling ratings were significantly higher in the $\mathrm{HE}$ conditions than in the LE conditions $\left(F(1,43)=19 \cdot 68, P<0 \cdot 001, \eta^{2}=0.31\right)$, but did not differ depending on sensory characteristics. At the 1-month follow-up, expected filling ratings still depended on energy content $\left(F(1,38)=8 \cdot 66, P=0 \cdot 006, \eta^{2}=0 \cdot 19\right)$, but not on sensory characteristics.

\section{Rated appetite}

Initial analyses confirmed no significant effects of test day, drink's energy content or sensory characteristics on baseline hunger and fullness ratings, and so the data were converted to changes from pre-drink ratings. As expected, hunger decreased immediately after ingestion and then recovering over the $90 \mathrm{~min}$ before lunch (main effect of time: $F(1,43)=198 \cdot 23, \quad P<0.001, \quad \eta^{2}=0 \cdot 82 ;$ Table 2$)$. However, these changes depended on test day, and the sensory characteristics and energy content of the drink, with significant interactions between sensory characteristics and test day

Table 2. Changes in rated hunger and fullness both immediately after consuming the drink (post-drink) and before the test meal (pre-lunch) before (pre-exposure, day 1), after the four exposure days (post-exposure, day 6) and 1 month later (follow-up, day 7 ) in the four drink conditions ${ }^{*}$

(Mean values with their standard errors)

\begin{tabular}{|c|c|c|c|c|c|c|c|c|c|c|c|c|c|}
\hline \multirow[b]{4}{*}{ Ratings } & \multirow[b]{4}{*}{ Drink conditions } & \multicolumn{12}{|c|}{ Day of study and time of rating } \\
\hline & & \multicolumn{4}{|c|}{ Pre-exposure } & \multicolumn{4}{|c|}{ Post-exposure } & \multicolumn{4}{|c|}{ Follow-up } \\
\hline & & \multicolumn{2}{|c|}{ Post-drink } & \multicolumn{2}{|c|}{ Pre-lunch } & \multicolumn{2}{|c|}{ Post-drink } & \multicolumn{2}{|c|}{ Pre-lunch } & \multicolumn{2}{|c|}{ Post-drink } & \multicolumn{2}{|c|}{ Pre-lunch } \\
\hline & & Mean & SEM & Mean & SEM & Mean & SEM & Mean & SEM & Mean & SEM & Mean & SEM \\
\hline \multirow{4}{*}{ Hunger } & LE/LS & -8 & 5 & 21 & 4 & -19 & 8 & 12 & 6 & -20 & 6 & 18 & 4 \\
\hline & LE/ES & -21 & 7 & 22 & 5 & -14 & 9 & 29 & 7 & -18 & 6 & 14 & 7 \\
\hline & $\mathrm{HE} / \mathrm{LS}$ & -16 & 6 & 17 & 5 & -17 & 5 & 9 & 5 & -17 & 8 & 12 & 7 \\
\hline & $\mathrm{HE} / \mathrm{ES}$ & -28 & 4 & 7 & 5 & -19 & 4 & 8 & 3 & -14 & 5 & 9 & 6 \\
\hline \multirow[t]{4}{*}{ Fullness } & LE/LS & 14 & 5 & -15 & 7 & 27 & 6 & -10 & 5 & 27 & 8 & -9 & 5 \\
\hline & LE/ES & 28 & 5 & -12 & 4 & 24 & 7 & -19 & 6 & 25 & 8 & -10 & 7 \\
\hline & HE/LS & 18 & 4 & -13 & 6 & 26 & 7 & -7 & 3 & 30 & 8 & -5 & 8 \\
\hline & $\mathrm{HE} / \mathrm{ES}$ & 26 & 4 & 4 & 5 & 20 & 7 & -5 & 4 & 30 & 6 & -5 & 3 \\
\hline
\end{tabular}

LE, low energy; HE, high energy; LS, low sensory; ES, enhanced sensory.

* Pre- and post-exposure, $n$ 12; follow-up LE/LS, $n$ 11; follow-up LE/ES, $n$ 10; follow-up HE/LS, $n 11$; follow-up HE/ES, $n 11$. 
$\left(F(1,43)=7 \cdot 72, P=0.008, \eta^{2}=0 \cdot 15\right)$ and between time and energy content $\left(F(1,43)=5 \cdot 29, P=0.026, \eta^{2}=0 \cdot 12\right)$. At the pre-exposure session, hunger decreased more immediately after consuming the ES drinks than the LS drinks $\left(F(1,43)=4.78, P=0.034, \eta^{2}=0 \cdot 10\right)$, and although hunger then increased by lunch, it increased less in the HE conditions than in the LE conditions $(F(1,43)=4 \cdot 29, P=0.044$, $\eta^{2}=0.09$ ), with the lowest increase in the HE/ES condition. There was no significant difference between the conditions in relation to hunger change immediately after consuming the drink, but these ratings differed immediately before lunch, with a significant effect of energy content $(F(1,43)=$ $\left.4.32, P=0.044, \eta^{2}=0.09\right)$, a marginal effect of sensory characteristics $\left(F(1,43)=3.46, P=0.07, \eta^{2}=0.07\right)$ and a marginal sensory characteristic $\times$ energy content interaction $(F(1,43)=$ 2.96, $P=0.09, \eta^{2}=0.06$ ), with hunger significantly greater in the LE/ES condition than in the other three conditions, which were similar. The analysis of changes in hunger at follow-up found no significant effects, although the data pattern (Table 2) was consistent with a sustained ability of the HE/ES combination to suppress hunger post-ingestion, which was masked by reduced power due to participant dropout.

The ratings of fullness tended to mirror hunger ratings (Table 2), with increased fullness immediately after consuming the drink and then recovery up to lunch $(F(1,43)=203 \cdot 51$, $\left.P<0.001, \eta^{2}=0.82\right)$. Although the change in fullness did not vary across days $\left(F(1,43)=0.04, P=0.85, \quad \eta^{2}=0.01\right)$, this depended both on sensory characteristics (test day $X$ sensory characteristic interaction: $F(1,43)=4 \cdot 10, P=0 \cdot 0491$, $\left.\eta^{2}=0.08\right)$ and energy content (test day $\times$ time $\times$ energy content: $\left.F(1,43)=5.55, P=0.023, \eta^{2}=0.11\right)$ of the test drink. At the pre-exposure session, the increase in fullness immediately after drink consumption depended on sensory characteristics $\left(F(1,43)=6 \cdot 27, P=0 \cdot 016, \eta^{2}=0 \cdot 13\right)$, with a larger increase in fullness after the ES versions than after the LS versions, but was not affected significantly by energy content. Immediately before lunch, fullness had decreased in all the conditions except the HE/ES condition, although data variability meant that the effects of condition were marginal $(F(3,43)=2 \cdot 31$, $\left.P=0.09, \eta^{2}=0 \cdot 14\right)$. In contrast, at the post-exposure session (day 6), fullness increased similarly in all the conditions immediately after consumption, but fullness tended to be lower after the LE conditions than after the HE conditions just before lunch $\left(F(1,43)=3 \cdot 82, P=0.057, \eta^{2}=0 \cdot 08\right)$, and a similar pattern was seen at the 1-month follow-up.

\section{Evaluations of drink preloads}

The drinks were designed so that the ES versions had a thicker texture and a more creamy flavour than the LS versions, and to confirm that these ratings of thick and creamy on days 1-6 were contrasted. These analyses confirmed that the ES versions of the drink were rated as more thick ( 72 (SEM 3)) and creamy (73 (SEM 2)) than the LS versions (thick: 56 (SEM 3) and creamy: 63 (SEM 2); thick: $F(1,42)=18.90, \quad P<0.001$, $\eta^{2}=0.31$ and creamy: $\left.F(1,42)=8.40, P=0.006, \eta^{2}=0 \cdot 17\right)$ No other effects were significant. Importantly, drinks were

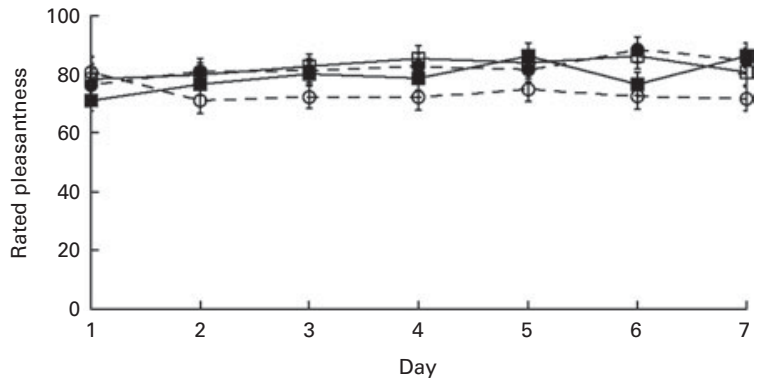

Fig. 3. Pleasantness of the test drinks across the seven test days (preexposure, day $1 ; 4 \mathrm{~d}$ of exposure, days $2-5$; post-exposure, day 6 ; follow-up, day 7 ) in the four drink conditions (LE, low energy; HE, high energy; LS, low sensory; ES, enhanced sensory). Values are means, with their standard errors represented by vertical bars. Pre- and post-exposure, $n 12$; follow-up LE/LS (-๑), $n$ 11; follow-up LE/ES (-๑), $n$ 10; follow-up HE/LS ( $\square), n 11$; follow-up HE/ES (--), $n 11$.

matched across energy content, and sensory characteristics did not change with exposure.

Rated pleasantness increased significantly across days 1-6 (linear contrast of test day: $F(1,42)=4 \cdot 60, P=0 \cdot 037, \eta^{2}=0 \cdot 10$ ), but these changes did not differ significantly between drink energy content or sensory conditions (Fig. 3).

\section{Discussion}

The present study suggests that a drink's nutrient content and sensory characteristics can both have an impact on satiety, but that repeated consumption changes the relative influence of these two drink aspects. Higher-energy drinks generated much stronger satiety than did low-energy drinks, and this effect was most pronounced in the high-energy drink with ES characteristics, though repeated consumption diminished this sensory effect. The low-energy versions of the drinks had weak effects on satiety and repeated consumption served to magnify this effect, particularly in the thinner less creamy versions of these drinks.

The key aim of the present study was to evaluate whether sensory-enhanced satiety was modified by repeated consumption. Consequently, it was important that sensory-enhanced satiety was evident before exposure, and analysis of data from day 1 confirmed this was so. Thus, the strongest satiety, indicated by reduced lunch intake and increased rated satiety (decreased hunger/increased fullness), was seen in the HE/ES condition, and the pattern of data from these between-participant contrasts was similar to that reported previously using within-participant designs ${ }^{(7,8)}$. However, while the HE/ES condition continued to generate the strongest satiety after repeated consumption, the difference between the HE/ES and HE/LS conditions decreased with repeated consumption. The largest effects of repeated consumption, however, was for the LE/LS drink, which generated weaker satiety after repeated consumption with significantly increased intake at the test meal both immediately after the exposure period and at the 1-month follow-up.

The present study also tested whether repeated consumption modified expectations about satiation and satiety. When the ratings of how filling participants expected the drinks to 
be (interpreted as expected satiation) were analysed, there was clear evidence that repeated exposure altered their perceptions. Thus, before exposure, expected satiation was determined solely by sensory characteristics: both the ES versions were rated as more filling than the LS ones regardless of energy content. However, over time, expected satiation increased for both the HE drinks, and decreased for the LE/ES drink, so that after the exposure period, this measure reflected energy content rather than sensory characteristics, and this effect was still evident at the 1-month follow-up. These data suggest that participants learned about the relative satiating effects of these products. The results from the ratings of how filling the product was expected to be are in line with an earlier finding that expected satiation increased after consumption of a higher-energy product ${ }^{(17)}$, although a subsequent study found no changes in a similar measure of expected satiation after repeated consumption ${ }^{(18)}$. The changes here in expected satiation were not observed for a measure of expected satiety based on the estimated portion of a breakfast cereal needed to suppress hunger to the same extent as the drink. As with expected satiation, before exposure, expected satiety varied with sensory characteristics, with higher expected satiety for the ES versions than for the LS versions regardless of nutrient content. However, despite clear changes in satiety responses to the different drinks, expected satiety measures did not change with repeated consumption. The difference between expected satiation and expected satiety measures might suggest that subtle changes in expectations about how satiating a product will be are not readily translated into estimates of how much of a different food would need to be consumed to generate the same level of satiety. Previously, we noted that responses to the two measures used here did not correlate significantly ${ }^{(10)}$, suggesting that they tapped into different aspects of expectations, although when expected satiety and expected satiation were both measured using portion size estimation the two measures were highly correlated (JM Brunstrom, unpublished results). Further research on the nature of these expectations is therefore needed.

It was predicted that the enhanced satiating effects of a thicker/creamier higher-energy drink would increase with repeated exposure through learned satiety. Since the effects of sensory manipulations in the high-energy drink were less evident after exposure and at the 1-month follow-up than at the start of the study (pre-exposure), the present study does not support the view that sensory manipulations can facilitate learned satiety. However, the largest changes in behaviour occurred with the low-energy drinks, and, in particular, repeated consumption of the LE/LS drink, where satiety became noticeably weaker over repeated consumption. The contrast of the effects of repeated consumption of the LE/LS and LE/ES drinks suggests that the presence of sensory characteristics that are associated with satiety (as evidenced by the higher expected satiety and filling measures for the LE/ES drink than the LE/LS drink) seemed to protect from overconsumption at lunch after a low-energy drink, suggesting that inclusion of the sensory characteristics that generate satiety expectations might limit learning about the lack of nutrients and be beneficial in the context of low-energy drink products. However, there was a tendency for the LE/ES drink to increase appetite and lunch intake when first encountered, an effect that has been noted in other studies (rebound hunger ${ }^{(7,8)}$ ), but which was not evident here after exposure. The change in expected filling with exposure could be interpreted as the evidence of learned satiety, with this evaluation changing as a consequence of exposure in line with the experience of actual satiety, although the lack of a similar effect with the expected satiety measure does limit this conclusion. Expected satiation has been shown to increase with familiarity ${ }^{(25)}$, although that study suggested that all foods tend to be expected to be more filling once they have been consumed repeatedly regardless of actual nutrient content, while the present data suggest that these changes are related to actual nutrient content.

One important feature of the present study was the inclusion of 1-month follow-up data, which clearly showed that the changes in response to the drinks immediately after exposure were maintained 1 month later despite any further experience of the drink. This suggests that the specific learning about the test products was robust, and suggests that learning that specific products are effective at suppressing appetite should lead to consistent and sustained improvements in appetite control.

In the present study, we manipulated both the thickness and creamy flavour of the drinks to generate the ES versions. Other data from our laboratory suggest that thickness manipulation is most likely to have an impact on behaviour ${ }^{(10)}$. However, thickness was manipulated by the addition of small amounts of tara gum, and an alternative explanation for the effects of this manipulation could be through a post-ingestive effect of the added tara gum. The addition of tara gum would have increased viscosity ${ }^{(10)}$, and viscosity has been reported to enhance satiation $^{(26)}$ and satiety ${ }^{(27,28)}$, perhaps by changing gastric emptying rate. However, the effects of sensory manipulations were ameliorated by repeated exposure, while the effects of added energy became more clear. Thus, even if the apparent effects of sensory manipulations could be explained by a post-ingestive effect, and various reasons suggest that this is unlikely ${ }^{(8)}$, any such effects are clearly modified by experience, suggesting that a simple post-ingestive effect of tara gum alone cannot readily explain the data.

It might have been predicted that repeated consumption of HE drinks would have led to increased liking for these products as a consequence of associations between their sensory characteristics and subsequent experience of satiety (flavournutrient learning ${ }^{(14,29)}$ ). Rated pleasantness increased similarly for all the four drinks. These results need to be interpreted with caution; however, as baseline liking was relatively high, limiting the scope for increased liking through exposure, and whether liking change is the best measure of flavournutrient learning, is questionable. Moreover, novelty is critical for flavour-nutrient learning ${ }^{(16)}$, and these products were not particularly novel. It would be therefore premature to consider the lack of liking change as the evidence against the concept of flavour-nutrient learning. In contrast, the changes in expected filling with exposure suggest that participants were 
learning about the consequences of consuming these products in support of flavour-nutrient learning. What aspect of nutrient detection underlies this effect cannot be determined from the present study, although animal studies have suggested that flavour-nutrient preference development is reinforced more by gut nutrient-sensing than post-ingestive use of nutrients ${ }^{(30)}$.

Overall, the present data confirm that in the short term, the satiating effects of a high-energy drink are modified by enhancing its satiety-relevant sensory characteristics, but that the effects of these sensory enhancements decrease, and the effects of its nutrients become more pronounced, following repeated consumption. The present data also suggest that drinks with minimal energy generate weak satiety and that repeated consumption of such drinks can lead to progressively weaker satiety responses, but that sensory modifications may help to ameliorate this effect.

\section{Acknowledgements}

The present study was funded by the BBSRC under the DRINC initiative (grant no. BB/H004645/1). The BBSRC had no role in the design, analysis or writing of this article.

M. R. Y. was the grant holder. L. C. developed the design with input from M. R. Y. and K. Mc. L. C. conducted the experimental work. M. R. Y. took the lead in analysing the data and preparing the manuscript. J. M. B. programmed the expected satiety task and made critical comments on the study design and the draft of the manuscript.

None of the authors had a conflict of interest with regard to conducting or reporting the study.

\section{References}

1. Hellstrom PM, Geliebter A, Naslund E, et al. (2004) Peripheral and central signals in the control of eating in normal, obese and binge-eating human subjects. Br J Nutr $\mathbf{9 2}$, S47-S57.

2. de Graaf C, Blom WA, Smeets PA, et al. (2004) Biomarkers of satiation and satiety. Am J Clin Nutr 79, 946-961.

3. Benelam B (2009) Satiation, satiety and their effects on eating behaviour. Nutr Bull 34, 126-173.

4. Mattes R (2006) Fluid calories and energy balance: the good, the bad, and the uncertain. Physiol Behav 89, 66-70.

5. Mattes R (2005) Soup and satiety. Physiol Behav 83, 739-747.

6. Bertenshaw EJ, Lluch A \& Yeomans MR (2013) Perceived thickness and creaminess modulates the short-term satiating effects of high-protein drinks. Br J Nutr 110, 578-586.

7. Chambers L, Ells H \& Yeomans MR (2013) Can the satiating power of a high energy beverage be improved by manipulating sensory characteristics and label information? Food Qual Prefer 28, 271-278.

8. Yeomans MR \& Chambers LC (2011) Satiety-relevant sensory qualities enhance the satiating effects of mixed carbohydrate-protein preloads. Am J Clin Nutr 94, 1410-1417.

9. Cassady BA, Considine RV \& Mattes RD (2012) Beverage consumption, appetite, and energy intake: what did you expect? Am J Clin Nutr 95, 587-593.
10. McCrickerd K, Chambers L, Brunstrom JM, et al. (2012) Subtle changes in the flavour and texture of a drink enhance expectations of satiety. Flavour 1, 20.

11. Crum AJ, Corbin WR, Brownell KD, et al. (2011) Mind over milkshakes: mindsets, not just nutrients, determine ghrelin response. Health Psychol 30, 424-429.

12. Booth DA (1987) Cognitive experimental psychology of appetite. In Eating Habits, pp. 175-209 [RA Boakes, MJ Burton and DA Popplewell, editors]. Chichester: Wiley.

13. Booth DA, Mather P \& Fuller J (1982) Starch content of ordinary foods associatively conditions human appetite and satiation, indexed by intake and pleasantness of starchpaired flavours. Appetite 3, 163-184.

14. Gibson EL \& Brunstrom JM (2007) Learned influences on appetite, food choice and intake: evidence in human beings. In Appetite and Body Weight: Integrative Systems and the Development of Anti-Obesity Drugs, pp. 271-300 [TC Kirkham and SJ Cooper, editors]. Burlington, MA: Academic Press.

15. Brunstrom JM (2011) The control of meal size in human subjects: a role for expected satiety, expected satiation and premeal planning. Proc Nutr Soc 70, 155-161.

16. Yeomans MR (2012) Flavour-nutrient learning in humans: an elusive phenomenon? Physiol Behav 106, 345-355.

17. Wilkinson LL \& Brunstrom JM (2009) Conditioning 'fullness expectations' in a novel dessert. Appetite 52, 780-783.

18. Hogenkamp PS, Brunstrom JM, Stafleu A, et al. (2012) Expected satiation after repeated consumption of low- or high-energy-dense soup. Br J Nutr 108, 182-190.

19. Mars M, Hogenkamp PS, Gosses AM, et al. (2009) Effect of viscosity on learned satiation. Physiol Behav 98, 60-66.

20. de Graaf C (2012) Texture and satiation: the role of orosensory exposure time. Physiol Behav 107, 496-501.

21. Stunkard AJ \& Messick S (1985) The three-factor eating questionnaire to measure dietary restraint, disinhibition and hunger. J Psychosom Res 29, 71-83.

22. Yeomans MR (2000) Rating changes over the course of meals: what do they tell us about motivation to eat? Neurosci Biobehav Rev 24, 249-259.

23. Brunstrom JM, Shakeshaft NG \& Scott-Samuel NE (2008) Measuring 'expected satiety' in a range of common foods using a method of constant stimuli. Appetite 51, 604-614.

24. Brunstrom JM \& Shakeshaft NG (2009) Measuring affective (liking) and non-affective (expected satiety) determinants of portion size and food reward. Appetite 52, 108-114.

25. Brunstrom JM, Shakeshaft NG \& Alexander E (2010) Familiarity changes expectations about fullness. Appetite $\mathbf{5 4}$, 587-590.

26. de Wijk RA, Zijlstra N, Mars M, et al. (2008) The effects of food viscosity on bite size, bite effort and food intake. Physiol Behav 95, 527-532.

27. Mattes RD \& Rothacker D (2001) Beverage viscosity is inversely related to postprandial hunger in humans. Physiol Behav 74, 551-557.

28. Zijlstra N, Mars M, de Wijk RA, et al. (2009) Effect of viscosity on appetite and gastro-intestinal hormones. Physiol Behav 97, 68-75.

29. Yeomans MR (2006) The role of learning in development of food preferences. In Psychology of Food Choice, pp. 93-112 [R Shepherd and M Raats, editors]. Wallingford, Oxford: CABI.

30. Sclafani A \& Ackroff K (2012) Role of gut nutrient sensing in stimulating appetite and conditioning food preferences. $A m J$ Physiol Regul, Integr Comp Physiol 302, R1119-R1133. 